\title{
Mapping the Topological Phase Diagram of Multiband Semiconductors with Supercurrents
}

\author{
Pablo San-Jose, ${ }^{1}$ Elsa Prada, ${ }^{2}$ and Ramón Aguado ${ }^{1}$ \\ ${ }^{1}$ Instituto de Ciencia de Materiales de Madrid, Consejo Superior de Investigaciones Científicas (ICMM-CSIC), \\ Sor Juana Inés de la Cruz 3, 28049 Madrid, Spain \\ ${ }^{2}$ Departamento de Física de la Materia Condensada, Instituto de Ciencia de Materiales Nicolás Cabrera \\ and Condensed Matter Physics Center (IFIMAC), Universidad Autónoma de Madrid, \\ Cantoblanco, 28049 Madrid, Spain
}

(Received 25 September 2013; published 4 April 2014)

\begin{abstract}
We show that Josephson junctions made of multiband semiconductors with strong spin-orbit coupling carry a critical supercurrent $I_{c}$ that contains information about the nontrivial topology of the system. In particular, we find that the emergence and annihilation of Majorana bound states in the junction is reflected in strong even-odd effects in $I_{c}$ at small junction transparency. This effect allows for a mapping between $I_{c}$ and the topological phase diagram of the junction, thus providing a dc measurement of its topology.
\end{abstract}

The broad appeal of topological materials lies in their gapped bulk surrounded by exotic edge states $[1,2]$. In topological superconductors, these edge states are Majorana fermions. A particularly interesting case arises when these Majorana fermions are zero-energy bound states [3]. Apart from their fundamental interest, these Majorana bound states (MBSs) are fascinating owing to their non-Abelian braiding properties [4] that have been proposed for fault-tolerant, topological, quantum computation. New possibilities to engineer topological superconductors that are more practical than early proposals based on exotic superconductors [4] have spurred great excitement. These are based on the proximity effect between a conventional $s$-wave superconductor and a topological insulator [5] or a semiconductor wire with strong spin-orbit (SO) coupling [6-10]. For the latter case, it has been shown $[9,10]$ that if an external Zeeman field $V_{Z}$, orthogonal to the $\mathrm{SO}$ axis, exceeds a critical value $V_{Z}^{c} \equiv \sqrt{\mu^{2}+\Delta^{2}}$, where $\mu$ is the Fermi energy and $\Delta$ is the induced $s$-wave pairing, zero-energy MBSs emerge at the wire's ends signaling a topologically nontrivial phase.

Recent experiments [11] in InSb nanowires have reported an emergent zero-bias anomaly (ZBA) in differential conductance $d I / d V$ as Zeeman field increases, which is consistent with the existence of zero-energy MBSs [12-15]. Subsequent experiments [16,17] show similar phenomenology, at least superficially. The question still stands, however, of whether these constitute true Majorana state detections, since other possible explanations, such as disorder [18-20], smooth confinement [15,21-23], or Kondo physics [24,25] (or the related 0.7 structure [26]) cannot be completely discarded. Recently, spin-split Andreev levels have been shown to lead to ZBAs similar to those expected for MBSs [27]. It has, thus, become urgent to study alternative "smoking gun" experiments.
One possibility is to consider topological Josephson junctions where the fusing of MBSs across the junction results in an odd number of Andreev level crossings at the Fermi energy when the superconducting phase advances by $2 \pi$. This is a manifestation of a nontrivial ground-state topology and translates into an anomalous $4 \pi$-periodic Josephson effect owing to fermionic parity conservation $[3,28,29]$. However, realistic effects that either spoil parity conservation (quasiparticle poisoning) or make the number of crossings even (finite length) translate into a $2 \pi$-periodic steady-state current [30-32]. Other dc transport observables, however, such as the dissipative multiple Andreev reflection current or the critical supercurrent $I_{c}$ retain strong signatures of the drastic changes that the Andreev bound states (ABSs) undergo across the topological transition in the single-band case [33]. It is, thus, natural to ask whether $I_{c}$ contains information about topology in the general multiband case.

Here, we demonstrate that the $I_{c}$ value of multimode junctions in the tunneling regime indeed provides a measure
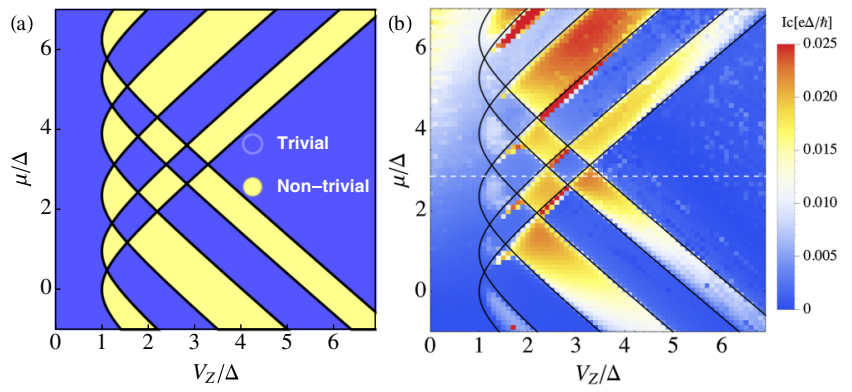

FIG. 1 (color online). (a) Topological phase diagram of a multimode semiconductor wire of width $W=2 l_{\mathrm{SO}}$, as a function of Zeeman splitting $V_{Z}$ and Fermi energy $\mu$. (b) Numerical computation of the critical supercurrent across a Josephson junction of small transparency made in such a wire. 
of topological order. In such a regime, $I_{c}\left(\mu, V_{Z}\right)$ maps the full topological phase diagram of the system; see Fig. 1. It exhibits strong even-odd effects, analogous to those predicted to occur in the spectrum of quasi-one-dimensional (quasi-1D) semiconductors containing near-zero MBSs owing to interband Rashba coupling [34-37]. We also quantify the resilience of the $I_{c}$ even-odd effect by considering a range of junction transparencies, finite wire length, and temperature.

Spectral even-odd effect.-The Bogoliubov-de Gennes Hamiltonian of a two-dimensional (2D) Rashba semiconductor reads

$$
\begin{aligned}
H= & \left(\frac{p^{2}}{2 m^{*}}-\mu\right) \tau_{z}+\frac{1}{\hbar}\left(\alpha_{x} \sigma_{y} p_{x} \tau_{z}-\alpha_{y} \sigma_{x} p_{y}\right) \\
& +\Delta \sigma_{y} \tau_{y}+V_{Z} \sigma_{x} \tau_{z},
\end{aligned}
$$

where $p^{2}=p_{x}^{2}+p_{y}^{2}, m^{*}$ is the effective mass, $\sigma_{i}$ are the spin Pauli matrices, and $\tau_{i}$ are Pauli matrices in the electron-hole sector. The last two terms describe an induced superconducting pairing of strength $\Delta$ and the Zeeman energy produced by an external magnetic field. For pure Rashba SO coupling, the two couplings $\alpha_{x, y}$ are equal, $\alpha_{x}=\alpha_{y}=\alpha_{\mathrm{SO}}$. For the sake of discussion, we also consider the possibility that they are different. It has been shown $[8,38]$ that the $s$-wave pairing in Eq. (1) induces both an effective $p_{x} \pm i p_{y}$ intraband pairing and an interband $s$-wave pairing when reexpressed in terms of the \pm eigenbasis of the helical Rashba + Zeeman normal problem. In this new language, a clear physical picture emerges: if $\mu$ and $V_{Z}$ are such that both $p_{x}+i p_{y}$ and $p_{x}-i p_{y}$ pairings occur, the system is topologically trivial. In contrast, if $V_{Z}$ exceeds the critical $V_{Z}^{c}$ value, only one of the two $p$-wave pairings $\Delta_{p}$ develops. The system then becomes topologically nontrivial.

In a quasi-1D geometry (with discrete confinement subbands), this picture is replicated for each subband independently (each has its own critical $V_{Z}^{(n)}=\sqrt{\Delta^{2}+\mu_{n}^{2}}$, with $\mu_{n}$ the Fermi energy measured from the bottom of the subband). If subbands are not coupled, each will contribute, above its critical $V_{Z}^{(n)}$ value, a single zero-energy MBS at each edge. If the subbands are coupled, e.g., through the transverse SO coupling $\alpha_{y}$, the $N$ MBSs per edge hybridize in pairs and form $\lfloor N / 2\rfloor$ full fermions at nonzero energies $\epsilon_{0}^{(n)}$ (with $n=1, \ldots,\lfloor N / 2\rfloor$ ) localized at each edge. (We denote the corresponding hole levels by $\epsilon_{0}^{(-n)}=-\epsilon_{0}^{(n)}$.) The remaining $N$ mod 2 end states stay as Majorana zero modes, at $\epsilon_{0}^{(0)}=0$ [34-36]. More formally, the subband coupling destroys the chiral symmetry $\left(\tau_{x} H \tau_{x}=-H\right)$ of the Hamiltonian, reducing its topological invariant from $\mathbb{Z}$ (BDI class in $1 D$ ) to $\mathbb{Z}_{2}$ (D class in 1D) [39-41]. This invariant has the meaning of the number of zero-energy bound states at the ends of the quasi1D semiconductor (arbitrary $N$, or $N \bmod 2$ ). This spectral even-odd effect is, thus, a manifestation of changes in the topological order in the multiband system.
To quantify the spectral even-odd effect, we analyze the local density of states (LDOS) at the end of a single multimode wire described by Eq. (1). We consider a finite value of $W / l_{\text {SO }}=2$ [42] but take the length of the wire $L_{S}$ to infinity so as to avoid complications arising from the overlap of Majorana states at opposite edges. The result, for fixed Fermi energy $\mu=2.8 \Delta$ [see dotted line in Fig. 1(b)], as a function of the Zeeman field $V_{Z}$, is shown in Fig. 2(a). It has been computed within a tight-binding discretization of Eq. (1) using a standard recursive Green's function approach [43]. At critical fields $V_{Z}^{(n)}$ each subband becomes topologically nontrivial. These points are visible by a closing of the superconducting gap (light gray), with an accompanying emergence of zero modes for odd transitions (nontrivial $\mathbb{Z}_{2}$ topological order of the wire). The remaining Majorana states are split to higher energies $\epsilon_{0}^{(n)}$. These appear as sharp resonances in Fig. 2(a). The odd phases have a zero-energy Majorana state $\epsilon_{0}^{(0)}=0$, whereas $n=0$ is missing in the even phases. This phenomenology is replicated throughout the $V_{Z}-\mu$ plane, resulting in the topological phase diagram shown in Fig. 1(a) [34-36].

Andreev levels in multiband junctions.-Next, we consider a Josephson junction between two segments in a multimode wire, with a superconducting phase difference $\phi$. Each segment is assumed long (large but finite $L_{S}$ ), but the junction is assumed short (normal length $L_{N} \rightarrow 0$ ), and of a given normal transparency per mode $T_{N}$. If the two segments are decoupled $\left(T_{N}=0\right)$, the previous discussion applies to each of them. Subgap states of energy $\epsilon_{0}^{(n)}$ form, localized at both the "inner" (at the junction) and "outer" ends of each segment. For finite $T_{N}$, eigenstates in each segment hybridize across the junction, and their energies $E^{(n)}(\phi)$ become $\phi$ dependent. These states are of three types. First, there are states above the gap that are delocalized across the wire and form a continuum or a dense quasicontinuum of levels $E_{\mathrm{qc}}^{(n)}(\phi)$ if the wire is of finite length. Second, a discrete set of conventional Andreev subgap levels $E_{\mathrm{cnv}}^{(n)}(\phi)$ form at the junction, two (in general Zeeman split) for each of the subbands that have not transitioned into their topological phase. Last, there are pairs of ABSs of topological origin $E_{\text {top }}^{(n, \pm)}(\phi)$, resulting from the bonding (-) and antibonding $(+)$ hybridization of each subgap $\epsilon_{0}^{(n)}$ on each side of the junction. (For $n=0, \pm$ is also the ground-state parity.) Their $\phi$ dependence at small $T_{N}$ values takes a simple form, $E_{\text {top }}^{(n, \pm)}(\phi) \approx \epsilon_{0}^{(n)} \pm$ $\delta E_{\text {top }}^{(n)} \cos (\phi / 2)$ [28,29]. Note that for large enough $L_{S}$ values, the $\epsilon_{0}^{(n)}$ subgap states localized at the outer ends of the wire remain unchanged when increasing the junction transparency. We compute all these $E^{(n)}(\phi)$ levels numerically by exact diagonalization of a discretized Eq. (1) and show them in Figs. 2(b) and 2(c) for a small $T_{N}$. In the even phase [panel (b)], only $n= \pm 1, \pm 2, \ldots$. ABSs of 

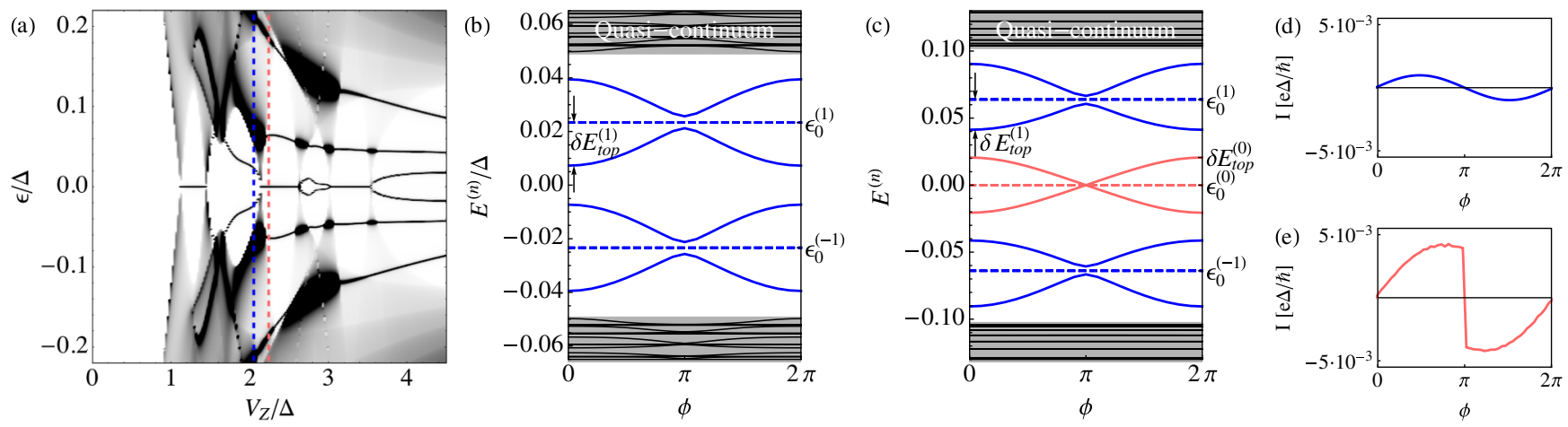

FIG. 2 (color online). (a) Local density of states at the end of a multimode wire for $\mu=2.8 \Delta$ [dashed line in Fig. 1(b)] and width $W=2 l_{\text {So }}$. White is zero, and black is maximum. The red (light) and blue (dark) dashed lines mark the Zeeman fields $V_{Z}=2.05 \Delta$ and $V_{Z}=2.25 \Delta$ used in panels (b) and (c), respectively. [(b), (c)] Andreev energy levels of a multimode Josephson junction as a function of phase difference $\phi$ in a topologically trivial $N=2$ even phase (b) and a nontrivial $N=3$ odd phase (c). The subgap levels $\epsilon_{0}^{(n)}$ at the inner ends of the two wire segments hybridize across the junction into $\phi$-dependent ABSs of topological origin [red (light) and blue (dark) solid lines] of amplitude $\delta E_{\text {top }}^{(n)}$, while those localized at the outer ends remain $\phi$ independent for large wire length $L_{S}$ values (dashed lines). Josephson supercurrents $I(\phi)$ associated to (b) and (c) are shown in (d) and (e), respectively, for $T_{N}=0.001$, including 200 levels into the quasicontinuum.

topological origin are present [blue (dark) curves]. In the odd phase [panel (c)], an additional $n=0$ ABS appears [red (light) curves] due to the hybridization of the Majorana zero modes. The $\phi=\pi$ crossing is only protected for this $n=0$ value. The $\phi$-independent levels at energies $\epsilon_{0}^{(n)}$ (dashed lines) correspond to Majorana states in the outer ends of the segments, whereas the rest of weakly $\phi$ dependent levels are either the $E_{\mathrm{cnv}}^{(n)}$ or $E_{\mathrm{qc}}^{(n)}$, whose variation with $\phi$ vanishes as $T_{N}$.

The Majorana hybridization across the junction was shown, in the case of a single mode, to be $\delta E_{\text {top }}^{(0)}=\Delta \sqrt{T_{N}}$ $[28,29]$. In the multimode case, level repulsion makes the $\delta E_{\text {top }}^{(n)}$ somewhat smaller and weakly $n$ dependent, but it remains true that $\delta E_{\text {top }}^{(n)} \propto \sqrt{T_{N}}$ as $T_{N} \rightarrow 0$. Hence, in this limit, the $\phi$ amplitude of the topological $E_{\text {top }}^{(n, \pm)}(\phi)$ vanishes much more slowly than that for conventional levels $E_{\text {cnv, qc }}^{(n)}(\phi)$. On the other hand, their average $\epsilon_{0}^{(n)}$ value depends on the degree of subband mixing that may be controlled via the ratio $W / l_{\text {SO }}$ if the dominant source of mixing is $\alpha_{y}$. The ratio $\delta E_{\mathrm{top}}^{(1)} / \epsilon_{0}^{(1)}$, which will become relevant below, can, therefore, be tuned through the junction width and its transparency. We now show how, depending on this ratio, an analogue of the spectral evenodd effect may develop in the junction's $I_{c}$.

Even-odd effect in the critical supercurrent.-We consider the $I_{c}$ of the junction and how it is affected by the system's topology. A Josephson junction supports a supercurrent that is in general given by [44]

$$
I(\phi)=-\frac{1}{2} \frac{e}{\hbar} \sum_{n} \tanh \left[\frac{E^{(n)}(\phi)}{2 k_{B} T}\right] \partial_{\phi} E^{(n)}(\phi),
$$

where the sum over levels $E^{(n)}$ includes both the standard $E_{\mathrm{cnv}, \mathrm{qc}}^{(n)}$ and the $E_{\mathrm{top}}^{(n, \pm)}$ of topological origin. In the tunneling limit, the sum is dominated by the latter, while the former, due to their suppressed $\phi$ derivative, contribute with a weak and featureless background, even across topological transitions.

Typical Josephson currents $I(\phi)$ in the tunneling limit for even and odd phases are shown in Figs. 2(d) and 2(e). Note that although both are $2 \pi$ periodic [30-32], phases with nontrivial topology exhibit a sawtooth $I(\phi)$ profile at small transparencies; panel (e). The critical current $I_{c}$ is given by the maximum of $|I(\phi)|$. If $\delta E_{\text {top }}^{(1)}<\epsilon_{0}^{(1)}$, then $\delta E_{\text {top }}^{(n)}<\epsilon_{0}^{(n)}$ for all $n>0$. In this regime, the zero-temperature contribution to $I(\phi)$ and, therefore, to $I_{c}$, of $E_{\mathrm{top}}^{(n,+)}$ and $E_{\mathrm{top}}^{(n,-)}$ will approximately cancel (to order $\sqrt{T_{N}}$ ) for any $n>0$ and $\phi$ (they have opposite $\phi$ derivatives and remain of constant sign for all $\phi$ values). However, the $n=0$ Majorana ABS, if present, does not cancel due to the sign change of the tanh function at zero energy. This extra contribution produces a $\sim \sqrt{T_{N}}$ increase in $I_{c}$ in odd phases,

$I_{c} \approx I_{c}^{\mathrm{bg}}+\left\{\begin{array}{ll}0 & \text { if } N \bmod 2=0 \\ \frac{1}{2} \frac{e}{\hbar} \delta E_{\mathrm{top}}^{(0)} & \text { if } N \bmod 2=1\end{array}\right.$ (for $\left.\delta E_{\mathrm{top}}^{(1)}<\epsilon_{0}^{(1)}\right)$,

where the background $I_{c}^{\mathrm{bg}}$ from conventional ABSs and quasicontinuum levels $E_{\text {cnv,qc }}^{(n)}$ is small $\left(\sim T_{N}\right)$ and independent of the system's $\mathbb{Z}_{2}$ topological invariant $N \bmod 2$. The $I_{c}$ even-odd contrast grows like $T_{N}^{-1 / 2}$ as $T_{N} \rightarrow 0$.

The result above suggests that a strong signature of the spectral even-odd effect should be visible in $I_{c}$ at small $T_{N}$ values. This is confirmed by the numerical simulation shown in Fig. 1(b), where the zero-temperature map 

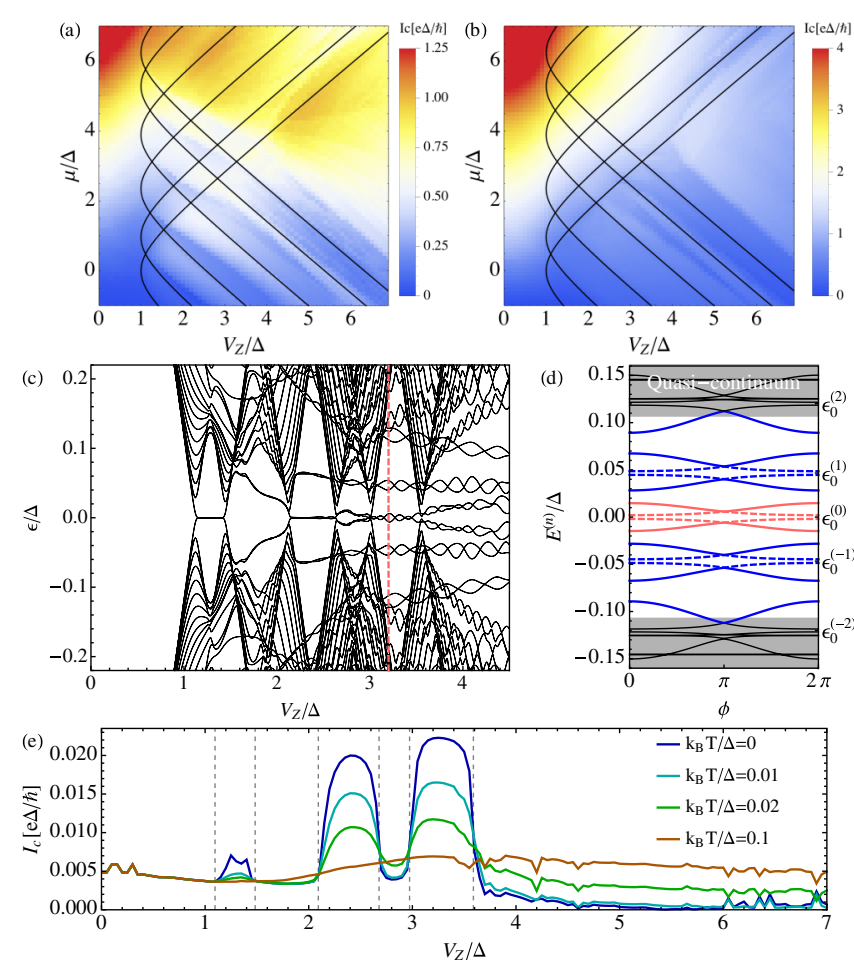

FIG. 3 (color online). [(a), (b)] Zero-temperature $I_{c}$ like that in Fig. 1(b) for $T_{N}=0.5$ (a) and $T_{N}=1$ (b). (c) Spectrum of a single wire like that of Fig. 2(a) but with a finite length $L_{S}=8.7 \mathrm{~W}$. (d) Corresponding ABS spectrum at $V_{Z}=3.2 \Delta$ [dashed line in (c)] at $T_{N}=0.005$. (e) Cut of the $I_{c}$ map of Fig. 1(b) for $\mu=2.8 \Delta$ at different temperatures.

$I_{c}\left(\mu, V_{Z}\right)$ is computed with $200 \mathrm{ABSs}$ (convergence achieved), obtained by exact diagonalization of a sixsubband Josephson junction with $W / l_{\text {SO }}=2$ and $T_{N}=$ 0.005 such that $\delta E_{\text {top }}^{(1)}<\epsilon_{0}^{(1)}$ [45]. Critical fields $V_{Z}^{(n)}$ are marked with black lines. Note that a large contrast in $I_{c}$ is visible between trivial and nontrivial phases. A cut for $\mu=2.8 \Delta$ is shown in Fig. 3(e), blue (upper) curve.

A number of factors suppress this correspondence between $I_{c}$ and topology. The most immediate are a high normal transparency of the junction (which decreases the contrast and increases the amplitude $\delta E_{\text {top }}^{(1)}$ ) and a smaller width (which decreases $\epsilon_{0}^{(1)}$ ). As soon as $\delta E_{\text {top }}^{(n)}>\epsilon_{0}^{(n)}$, the cancellation of supercurrents from $E_{\text {top }}^{(n,+)}$ and $E_{\text {top }}^{(n,-)}$ becomes restricted to a finite range around $\phi=\pi$, where the sign of the tanh is constant. Outside of this range, the two levels will add instead of cancel, and their contribution to $I_{c}$ will become finite even for $N \bmod 2=0$. The resulting loss of topological contrast is illustrated in Figs. 3(a) and 3(b), where the same map of Fig. 1(b) is presented for $T_{N}=0.5$ and $T_{N}=1$, respectively.

Another potentially deleterious factor to the $I_{c}$ even-odd effect is the finite length $L_{S}$ of the two superconducting segments of the wire. In a single decoupled wire, the MBSs at each end decay into the bulk within a typical localization length [46] of the order of the coherence length of each subband $\xi_{n} \approx \hbar v_{F}^{(n)} / \Delta$ (where $v_{F}^{(n)}$ is the corresponding Fermi velocity). When $L_{S} \lesssim \max _{n} \xi_{n}$, the Majorana states of different ends overlap. This modifies the spectral even-odd structure, since zero modes at different ends in an odd phase hybridize into a fermion of finite energy that oscillates with $V_{Z}[15,22,47,48]$. Figure 3(c) shows the spectrum of a wire like that in Fig. 2(a) but with $L_{S}=8.7 \mathrm{~W}$. Note, however, that while a finite $L_{S}$ leads to zero-mode splittings at high fields $V_{Z}$, those at smaller fields are typically much more robust, owing to their smaller Fermi velocities (which grow with $V_{Z}$ ) and, hence, smaller localization lengths. In a Josephson junction geometry, a finite $L_{S}$ will likewise lead to the hybridization of outer- and inner-edge zero modes; see Fig. 3(d). This suppresses their contribution to the supercurrent and, hence, the even-odd contrast in $I_{c}$ but typically only for high $V_{Z}$ values like those in panel (c). Note, moreover, that even though the spectrum in Fig. 3(d) corresponds to an odd phase, it is actually trivial for finite $L_{S}$, rigorously speaking [49], since it has an even number of zero-energy crossings as a function of $\phi$ [50].

Finally, we consider the effect of finite temperature. Figure 3(e) shows a cut of the $I_{c}$ map of Fig. 1(b) for $\mu=$ $2.8 \Delta$ and four different temperatures. Vertical dashed lines mark the critical fields $V_{Z}^{(n)}$. Note that $I_{c}$ exhibits a smooth background offset $I_{c}^{\mathrm{bg}}$, with comparatively large plateaus superimposed in odd phases [see the blue (upper) curve for $T=0$ ]. Increasing temperatures above $\epsilon_{0}^{(1)}$ quickly washes out the even-odd contrast. The energy $\epsilon_{0}^{(1)}$ [around $0.07 \Delta$ in this example, see Fig. 2(c)] is sometimes called the minigap [36] or the energy scale protecting Majorana physics, and, consequently, the $I_{c}$ even-odd effect.

In conclusion, we have shown that the critical supercurrent of a multimode Josephson junction in a quasi-1D Rashba semiconductor wire with induced superconductivity can be used to directly measure the $\mathbb{Z}_{2}$ topological invariant. This invariant gives the number-0 (trivial) or 1 (nontrivial) — of Majorana zero modes at each boundary in the wire. We found that, in topologically nontrivial phases, the $I_{c}$ value for small junction transparency is strongly enhanced relative to trivial phases by virtue of the additional supercurrent contributed by Majorana zero modes in the junction. It represents a transport analogue of the spectral even-odd effect in a uniform wire. Given the extremely clean and precise measurements of $I_{c}$ possible today [16,51-55], this effect could prove to be a useful route towards the detection of topological transitions and, hence, MBSs in these systems and an alternative to the ZBAs exploited to date. We have evaluated the impact of a range of factors that may mask the even-odd contrast and concluded that ballistic junctions currently available should exhibit topological signatures in $I_{c}$ at temperatures below a few hundreds of $\mathrm{mK}$ and small transparency. 
We gratefully acknowledge discussions with F. Giazotto, M. Amado, and A. Fornieri. This work was supported by the European Research Council and the Spanish Ministry of Economy and Innovation through Grants No. FIS201123713 (P. S.-J), No. FIS2012-33521 (R. A.), and No. FIS2010-21883 and the Ramón y Cajal Program (E. P.).

[1] X.-L. Qi and S.-C. Zhang, Rev. Mod. Phys. 83, 1057 (2011).

[2] M. Z. Hasan and C. L. Kane, Rev. Mod. Phys. 82, 3045 (2010).

[3] A. Y. Kitaev, Phys. Usp. 44, 131 (2001).

[4] N. Read and D. Green, Phys. Rev. B 61, 10267 (2000).

[5] L. Fu and C. L. Kane, Phys. Rev. Lett. 100, 096407 (2008).

[6] M. Sato, Y. Takahashi, and S. Fujimoto, Phys. Rev. Lett. 103, 020401 (2009).

[7] J. D. Sau, R. M. Lutchyn, S. Tewari, and S. Das Sarma, Phys. Rev. Lett. 104, 040502 (2010).

[8] J. Alicea, Phys. Rev. B 81, 125318 (2010).

[9] R. M. Lutchyn, J. D. Sau, and S. Das Sarma, Phys. Rev. Lett. 105, 077001 (2010).

[10] Y. Oreg, G. Refael, and F. von Oppen, Phys. Rev. Lett. 105, 177002 (2010).

[11] V. Mourik, K. Zuo, S. M. Frolov, S. R. Plissard, E. P. A. M. Bakkers, and L. P. Kouwenhoven, Science 336, 1003 (2012).

[12] K. Sengupta, I. Zutic, H.-J. Kwon, V. M. Yakovenko, and S. Das Sarma, Phys. Rev. B 63, 144531 (2001).

[13] C. J. Bolech and E. Demler, Phys. Rev. Lett. 98, 237002 (2007).

[14] K. T. Law, P. A. Lee, and T. K. Ng, Phys. Rev. Lett. 103, 237001 (2009).

[15] E. Prada, P. San-Jose, and R. Aguado, Phys. Rev. B 86, 180503(R) (2012).

[16] M. T. Deng, C. L. Yu, G. Y. Huang, M. Larsson, P. Caroff, and H. Q. Xu, Nano Lett. 12, 6414 (2012).

[17] A. Das, Y. Ronen, Y. Most, Y. Oreg, M. Heiblum, and H. Shtrikman, Nat. Phys. 8, 887 (2012).

[18] F. Pientka, G. Kells, A. Romito, P. W. Brouwer, and F. von Oppen, Phys. Rev. Lett. 109, 227006 (2012).

[19] D. Bagrets and A. Altland, Phys. Rev. Lett. 109, 227005 (2012).

[20] J. Liu, A. C. Potter, K. T. Law, and P. A. Lee, Phys. Rev. Lett. 109, 267002 (2012).

[21] T. D. Stanescu and S. Tewari, Phys. Rev. B 87, 140504 (2013).

[22] D. Rainis, L. Trifunovic, J. Klinovaja, and D. Loss, Phys. Rev. B 87, 024515 (2013).

[23] G. Kells, D. Meidan, and P. W. Brouwer, Phys. Rev. B 86, 100503 (2012).

[24] E. J. H. Lee, X. Jiang, R. Aguado, G. Katsaros, C. M. Lieber, and S. De Franceschi, Phys. Rev. Lett. 109, 186802 (2012).

[25] A. D. K. Finck, D. J. Van Harlingen, P. K. Mohseni, K. Jung, and X. Li, Phys. Rev. Lett. 110, 126406 (2013).

[26] H. O. H. Churchill, V. Fatemi, K. Grove-Rasmussen, M. T. Deng, P. Caroff, H. Q. Xu, and C. M. Marcus, Phys. Rev. B 87, 241401 (2013).

[27] E. J. H. Lee, X. Jiang, M. Houzet, R. Aguado, C. M. Lieber, and S. De Franceschi, Nat. Nanotechnol. 9, 79, (2014).

[28] L. Fu and C. L. Kane, Phys. Rev. B 79, 161408 (2009).
[29] H. Kwon, K. Sengupta, and V. Yakovenko, Eur. Phys. J. B 37, 349 (2003).

[30] D. M. Badiane, M. Houzet, and J. S. Meyer, Phys. Rev. Lett. 107, 177002 (2011).

[31] P. San-Jose, E. Prada, and R. Aguado, Phys. Rev. Lett. 108, 257001 (2012).

[32] D. I. Pikulin and Y. V. Nazarov, Phys. Rev. B 86, 140504 (2012).

[33] P. San-Jose, J. Cayao, E. Prada, and R. Aguado, New J. Phys. 15, 075019 (2013).

[34] R. M. Lutchyn, T. D. Stanescu, and S. Das Sarma, Phys. Rev. Lett. 106, 127001 (2011).

[35] A. C. Potter and P. A. Lee, Phys. Rev. B 83, 184520 (2011).

[36] T. D. Stanescu, R. M. Lutchyn, and S. Das Sarma, Phys. Rev. B 84, 144522 (2011).

[37] M. Gibertini, F. Taddei, M. Polini, and R. Fazio, Phys. Rev. B 85, 144525 (2012).

[38] S. Fujimoto, Phys. Rev. B 77, 220501 (2008).

[39] A. P. Schnyder, S. Ryu, A. Furusaki, and A. W. W. Ludwig, Phys. Rev. B 78, 195125 (2008).

[40] S. Tewari and J. D. Sau, Phys. Rev. Lett. 109, 150408 (2012).

[41] A. Altland and M. R. Zirnbauer, Phys. Rev. B 55, 1142 (1997).

[42] Here, $\alpha_{x}=\alpha_{y}$, so that $l_{\mathrm{so}}^{x}=l_{\mathrm{so}}^{y} \equiv l_{\mathrm{so}}$.

[43] S. Datta, Electronic Transport in Mesoscopic Systems (Cambridge University Press, Cambridge, England, 1997).

[44] C. Beenakker, in Transport Phenomena in Mesoscopic Systems: Proceedings of the 14th Taniguchi Symposium, Shima, Japan, November 10-14, 1991 (Springer-Verlag, Berlin, 1992).

[45] For the plots of Figs. 1(b) and 3(a) and 3(b) we have considered $\alpha_{x} \neq \alpha_{y}$, particularly $\alpha_{y} / \alpha_{x}=0.4$. We introduce this artificial asymmetry for computational reasons. With a stronger SO coupling in the $x$ direction, we can perform our numerical calculations with a shorter total length of the system such that the outer Majorana states remain decoupled from the junction, yielding flat ABSs as a function of $\phi$. It should be noted, however, that the even-odd effect we describe is independent of this asymmetry (as long as inner and outer Majorana states do not overlap).

[46] J. Klinovaja and D. Loss, Phys. Rev. B 86, 085408 (2012).

[47] J. S. Lim, L. Serra, R. López, and R. Aguado, Phys. Rev. B 86, 121103 (2012).

[48] S. Das Sarma, J. D. Sau, and T. D. Stanescu, Phys. Rev. B 86, 220506 (2012).

[49] D. I. Pikulin and Y. V. Nazarov, JETP Lett. 94, 752 (2011).

[50] C. W. J. Beenakker, J. M. Edge, J. P. Dahlhaus, D. I. Pikulin, S. Mi, and M. Wimmer, Phys. Rev. Lett. 111, 037001 (2013).

[51] M. Amado, A. Fornieri, F. Carillo, G. Biasiol, L. Sorba, V. Pellegrini, and F. Giazotto, Phys. Rev. B 87, 134506 (2013).

[52] Y.-J. Doh, J. A. van Dam, A. L. Roest, E. P. A. M. Bakkers, L. P. Kouwenhoven, and S. De Franceschi, Science 309, 272 (2005).

[53] T. Nishio, T. Kozakai, S. Amaha, M. Larsson, H. A. Nilsson, H. Q. Xu, G. Zhang, K. Tateno, H. Takayanagi, and K. Ishibashi, Nanotechnology 22, 445701 (2011).

[54] H. A. Nilsson, P. Samuelsson, P. Caroff, and H. Q. Xu, Nano Lett. 12, 228 (2012).

[55] H. Y. Günel, I. E. Batov, H. Hardtdegen, K. Sladek, A. Winden, K. Weis, G. Panaitov, D. Grützmacher, and T. Schäpers, J. Appl. Phys. 112, 034316 (2012). 